\title{
PRINCIPAL SPECIALIZATIONS OF SCHUBERT POLYNOMIALS AND PATTERN CONTAINMENT
}

\author{
YIBO GAO
}

\begin{abstract}
We show that the principal specialization of the Schubert polynomial at $w$ is bounded below by $1+p_{132}(w)+p_{1432}(w)$ where $p_{u}(w)$ is the number of occurrences of the pattern $u$ in $w$, strengthening a previous result by $\mathrm{A}$. Weigandt. We then make a conjecture relating the principal specialization of Schubert polynomials to pattern containment. Finally, we characterize permutations $w$ whose RC-graphs are connected by simple ladder moves via pattern avoidance.
\end{abstract}

\section{INTRODUCTION}

The study of principal specializations of Schubert polynomials $\nu_{w}:=\mathfrak{S}_{w}(1, \ldots, 1)$ has seen interesting results in recent years. Geometrically, $\nu_{w}$ equals the degree of the matrix Schubert variety [2] corresponding to $w$ and combinatorially, $\nu_{w}$ equals the number of RC-graphs of $w$. For a fixed $n \in \mathbb{Z}_{>0}$, Stanley [6] asked the question of determining the permutations $w \in S_{n}$ that achieve the maximum value of $\nu_{w}$ and it is conjectured by Merzon and Smirnov [4 that such $w$ must be layered. Assuming that $w$ is layered, Morales, Pak and Panova [5] determined such $w$ 's that achieve the maximum value of $\nu_{w}$ and the asymptotic behavior of this value.

Definition 1.1. For $u \in S_{k}$ and $w \in S_{n}$, define the number of occurrences of $u$ in $w$ to be

$$
\begin{gathered}
p_{u}(w):=\#\left\{1 \leq a_{1}<\cdots<a_{k} \leq n \mid w\left(a_{i}\right)<w\left(a_{j}\right) \text { if and only if } u(i)<u(j),\right. \\
\text { for all } 1 \leq i<j \leq k\} .
\end{gathered}
$$

We can extend Definition 1.1 to $w \in S_{\infty}$, permutations of $\mathbb{Z}_{>0}$ with all but a finite number of fixed points, as long as $u(k) \neq k$.

Weigandt [7] showed that $\nu_{w}$ is bounded below by $1+p_{132}(w)$, where $p_{132}(w)$ is the number of 132 patterns in $w$. However, this inequality is far from being tight. In this paper, we strengthen this inequality (Section 2) and provide a conjecture (Section 3) that strongly relates the principal specializations of Schubert polynomials $\nu_{w}$ to enumeration of pattern containment in $w$.

We start with some background on Schubert polynomials and RC-graphs. We refer readers to [1] and [3] for detailed exposition on this subject matter.

An $R C$-graph of $w$ is a finite subset $D \subset \mathbb{Z}_{>0} \times \mathbb{Z}_{>0}$ such that

$$
\prod_{i=1}^{\infty} \prod_{j=\infty,(i, j) \in D}^{1} s_{i+j-1}=w
$$

Date: October 22, 2019. 
is a reduced word for $w$. The set of RC-graphs of $w$ is denoted as $\mathcal{R C}(w)$. For an RC-graph $D$, its weight is defined to be $\operatorname{wt}(D)=\prod_{(i, j) \in D} x^{i}$. The following theorem is well-known.

Theorem $1.2([1])$. For a permutation $w \in S_{\infty}, \mathfrak{S}_{w}=\sum_{D \in \mathcal{R C}(w)} \operatorname{wt}(D)$.

As a result, the principal specialization of Schubert polynomials $\nu_{w}:=\mathfrak{S}_{w}(1, \ldots, 1)$ equals $\# \mathcal{R C}(w)$, the number of $\mathrm{RC}$-graphs for $w$, which is the quantity that we are interested in.

An RC-graph $D$ may be viewed as a strand diagram in the $2 \mathrm{D}$ grid $\mathbb{Z}_{>0}^{2}$ by placing a crossing + at each $(i, j) \in D$ and placing an elbow ${ }^{\prime}$ (or non-crossing) at each $(i, j) \notin D$. This procedure produces a pseudo-line arrangement that connects $(k, 0)$ with $(0, w(k))$ where no two strands intersect more than once where $D \in \mathcal{R C}(w)$. For a clearer view of the local moves, we are also going to denote a crossing by + and an elbow by $\cdot$. See Figure 1 for an example.
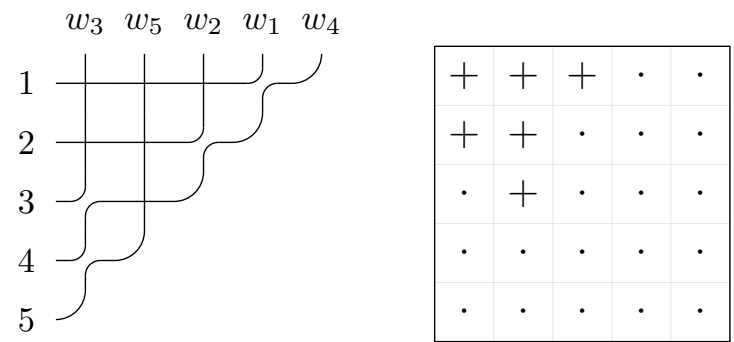

Figure 1. An RC-graph for $w=43152$

We label the strands such that strand $k$ starts from the left of row $k$ and ends at the top of column $w(k)$. For a crossing $(i, j) \in D$, we say that it has type $(a, b)$ if the two strands intersecting at $(i, j)$ are labeled with $a<b$. It is clear that all crossings of $D \in \mathcal{R C}(w)$ have different types and are in one-to-one correspondence with $\{(i, j) \mid i<j, w(i)>w(j)\}$, the inversions of $w$.

There are distinguished RC-graphs of $w$. Recall that the Rothe diagram of $w$ is the set

$$
R D(w):=\left\{(i, j) \mid 1 \leq i, j \leq n, w(i)>j, w^{-1}(j)>i\right\} .
$$

The bottom $R C$-graph of $w$ is the set of squares left-justified from $R D(w)$, which formally is $B_{w}:=\left\{(i, j) \mid j \leq \operatorname{code}(w)_{i}\right\}$ where $\operatorname{code}(w)$ is the Lehmar code of $w$ defined via code $(w)_{i}=\#\{j>i \mid w(j)<w(i)\}$. Similarly, the top RC-graph of $w$ is the set of sqaures top-adjusted from $R D(w)$, which is $T_{w}:=\{(i, j) \mid i \leq$ code $\left.\left(w^{-1}\right)_{j}\right\}$. An example is shown in Figure 2

Bergeron and Billey [1] described the following local moves on $\mathcal{R C}(w)$, which are called ladder moves.

Definition 1.3. A ladder move of $D \in \mathcal{R C}(w)$ at a crossing $(i, j) \in D$ of order $k \geq 0$ produces another RC-graph $D^{\prime}=D \backslash\{(i, j)\} \cup\{(i-k-1, j+1)\} \in \mathcal{R C}(w)$ if the following conditions on $D$ are satisfied:

(1) $(i, j) \in D,(i, j+1),(i-k-1, j),(i-k-1, j+1) \notin D$;

(2) for all $i-k \leq i^{\prime}<i,\left(i^{\prime}, j\right),\left(i^{\prime}, j+1\right) \in D$. 

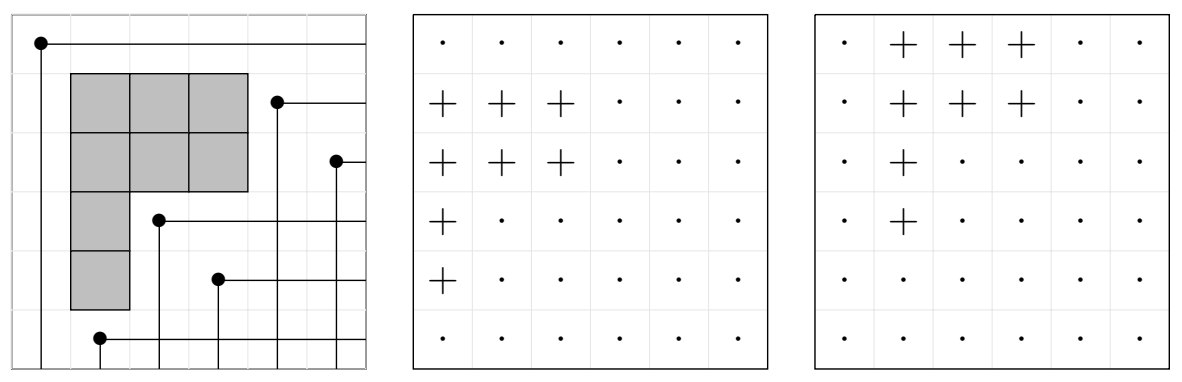

Figure 2. The Rothe diagram $R D(w)$, bottom RC-graph $B_{w}$ and top RC-graph $T_{w}$ for $w=156342$.

$$
k\left\{\begin{array}{cc}
\cdot & \cdot \\
+ & + \\
\vdots & \vdots \\
\vdots & \vdots \\
+ & + \\
+ & \cdot
\end{array} \quad \mapsto \quad \begin{array}{cc}
\cdot & + \\
+ & + \\
\vdots & \vdots \\
\vdots & \vdots \\
+ & + \\
\cdot & \cdot \\
\hline
\end{array}\right.
$$

Figure 3. A ladder move of order $k$.

It is straightforward to observe that such a ladder move preserves the permutation and the reducedness. Visually, a ladder move is depicted in Figure 3 .

Let $D \mapsto D^{\prime}$ be a ladder move that removes $(i, j) \in D$ and adds $(i-k-1, j+1) \in$ $D^{\prime}$. We see that if the crossing $(i, j) \in D$ is of type $(a, b)$, then $(i-k-1, j+1) \in D^{\prime}$ is of type $(a, b)$ as well, while all other crossings have the same type as before.

Theorem 1.4 (1]). Any $R C$-graph $D \in \mathcal{R C}(w)$ can be obtained from $B_{w}$ by a sequence of ladder moves.

We adopt the following definition from [7].

Definition 1.5. For $D \in \mathcal{R C}(w)$, let its label $\alpha(D)$ be a vector such that

$$
\alpha(D)_{k}:=\{(i, j) \in D \mid i+j-1=k\} .
$$

The label $\alpha(D)$ records the number of crossings on each diagonal. We say that a crossing $(i, j) \in D$ is on diagonal $i+j-1$ and denote it by $\operatorname{diag}((i, j))=i+j-1$.

A ladder move is called simple if its order is 0 , and a ladder move is non-simple if its order is positive. It is then clear that simple ladder moves do not change the label of RC-graphs, while non-simple ladder moves increase the label lexicographically.

\section{THE MAIN THEOREM}

Theorem 2.1. For $w \in S_{\infty}, \mathfrak{S}_{w}(1) \geq 1+p_{132}(w)+p_{1432}(w)$.

Theorem 2.1 strengthens the following theorem by A. Weigandt.

Theorem 2.2. [7] For $w \in S_{\infty}, \mathfrak{S}_{w}(1) \geq 1+p_{132}(w)$. 
Let's quickly review the proof idea of Theorem 2.2. Weigandt [7] showed that the top RC-graph $T_{w}$ and the bottom RC-graph $B_{w}$ are connected by a sequence of simple ladder moves and any such a chain of RC-graphs contains exactly $1+p_{132}(w)$ RC-graphs of $w$. Since simple ladder moves don't change the label of RC-graphs, to prove Theorem 2.1. it suffices to construct $p_{1432}(w)$ RC-graphs with a different label than $\alpha\left(B_{w}\right)$.

Example 2.3. Figure 4 shows the Rothe diagram and all RC-graphs of the permutation $w=1432$. We see that $\# \mathcal{R C}(w)=5$, while $1+p_{132}(w)=4$, accounting for the first 4 RC-graphs (from left to right) listed in Figure 4 . The left most RCgraph is $B_{w}$ and the fourth is $T_{w}$. These four RC-graphs can be obtained from the previous one by a simple ladder move, while the fifth RC-graph is obtained from $B_{w}$ by a ladder move of order 1 .
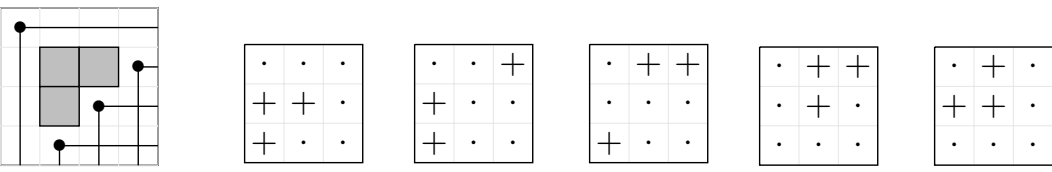

FiguRE 4. The Rothe diagram and all RC-graphs of $w=1432$.

Remark 2.4. It is not true that ladder moves of order 1 (together with simple ladder moves) can produce $p_{1432}(w)$ many RC-graphs. To prove Theorem 2.1] we have to utilize ladder moves of high orders. For example, if $w=14532$, then $p_{1432}(w)=2$. But starting from $B_{w}$ and applying any sequence of ladder moves of order 0 or 1 , we can only obtain one RC-graph (the right of Figure 5) that has a different label than $B_{w}$.
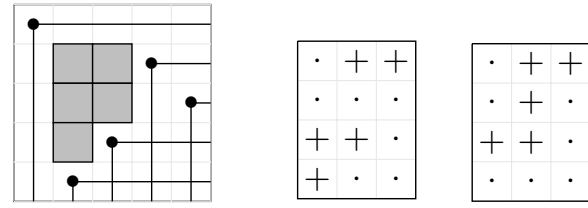

Figure 5. The Rothe diagram and the only ladder move of order 1 for $w=14532$.

For each $(i, j) \in R D(w)$, let $A_{(i, j)}:=\{a<i \mid w(a)<j\}$ and $C_{(i, j)}:=\left\{i<c<w^{-1}(j) \mid j<w(c)<w(i)\right\}$. Pictorially in the Rothe diagram, for the box $(i, j) \in R D(w), A_{(i, j)}$ is the set of dots (permutation entries) to the top left of $(i, j)$ and $C_{(i, j)}$ is the set of dots inside the rectangle to the bottom right of $(i, j)$ with vertices at $(i, w(i))$ and $\left(w^{-1}(j), j\right)$.

After left-justification, $(i, j) \in R D(w)$ becomes $\left(i, j-\# A_{(i, j)}\right) \in B_{w}$. Denote this map by $\beta: R D(w) \rightarrow B_{w}$. If $\left(i, j_{1}\right)$ and $\left(i, j_{2}\right)$, two boxes in the same row of $R D(w)$ with $j_{1}<j_{2}$, have no squares in between, then they become adjacent after left-justification in $B_{w}$. Notice that $\operatorname{diag}(\beta(i, j))=i+j-\# A_{(i, j)}-1$. This expression is symmetric in $i$ and $j$. If $\left(i_{1}, j\right)$ and $\left(i_{2}, j\right)$, two boxes in the same column of $R D(w)$ with $i_{1}<i_{2}$, have no squares in between, then we similarly have $\operatorname{diag}\left(\beta\left(i_{1}, j\right)\right)+1=\operatorname{diag}\left(\beta\left(i_{2}, j\right)\right)$. This is because $\# A_{\left(i_{2}, j\right)}-\# A_{\left(i_{1}, j\right)}$ must be $i_{2}-i_{1}-1$ for there to be no squares left in between of $\left(i_{1}, j\right)$ and $\left(i_{2}, j\right)$ in $R D(w)$. 
Lemma 2.5. Let $(i, j),\left(i^{\prime}, j^{\prime}\right) \in R D(w)$ such that $i<i^{\prime}$ and $j<j^{\prime}$, then $\operatorname{diag}\left(\beta\left(i^{\prime}, j^{\prime}\right)\right)-$ $\operatorname{diag}(\beta(i, j)) \geq 2$.

Proof. For $(i, j) \in R D(w), w(i)>j$ and $w^{-1}(j)>i$ so

$$
\begin{aligned}
\operatorname{diag}(\beta(i, j))= & i+j-\# A_{(i, j)}-1 \\
= & \#\{(a, w(a)) \mid a \leq i\}+\#\{(a, w(a)) \mid w(a) \leq j\} \\
& -\#\{(a, w(a)) \mid a<i, w(a)<j\}-1 \\
= & \#\{(a, w(a)) \mid a \leq i \text { or } w(a) \leq j\}-1 \\
= & n-1-\#\{(a, w(a)) \mid a>i, w(a)>j\} .
\end{aligned}
$$

If $i^{\prime}>i$ and $j^{\prime}>j$, then $\{(a, w(a)) \mid a>i, w(a)>j\}$ contains $\{(a, w(a)) \mid a>$ $\left.i^{\prime}, w(a)>j^{\prime}\right\}$ disjoint union with $\left\{(i, w(i)),\left(w^{-1}(j), j\right)\right\} \operatorname{so} \operatorname{diag}\left(\beta\left(i^{\prime}, j^{\prime}\right)\right)-\operatorname{diag}(\beta(i, j)) \geq$ 2 .

Intuitively, Lemma 2.5 is saying that if a box $\left(i^{\prime}, j^{\prime}\right)$ lies strictly to the bottom right of $(i, j)$ in $R D(w)$, then after left-justification to $B_{w}$, they won't interact with each other when applying simple ladder moves.

Proof of Theorem [2.1. Consider the following calculation

$$
\begin{aligned}
p_{1432}(w) & =\sum_{b<d, w(b)>w(d)} \#\{a<b \mid w(a)<w(d)\} \cdot \#\{b<c<d \mid w(d)<w(c)<w(b)\} \\
& =\sum_{(i, j) \in R D(w)} \#\{a<i \mid w(a)<j\} \cdot \#\left\{i<c<w^{-1}(j) \mid j<w(c)<w(i)\right\} .
\end{aligned}
$$

We first summarize our strategy. We will construct $\mathcal{P}_{(i, j)} \subset \mathcal{R C}(w)$, a set of RCgraphs of $w$ with different labels than that of $B_{w}$, whose cardinality is at least $\# A_{(i, j)} \cdot \# C_{(i, j)}$. We finish the proof by showing that such sets $\mathcal{P}_{(i, j)}$ don't intersect for distinct $(i, j) \in R D(w)$.

Now fix $(i, j) \in R D(w)$. Let $C_{(i, j)}$ be $\left\{c_{1}, \ldots, c_{q}\right\}$, ordered (from left to right) such that $j<w\left(c_{1}\right)<\cdots<w\left(c_{q}\right)<w(i)$. Notice that we don't have to do anything if $C_{(i, j)}=\emptyset$. The choice of $c_{1}$ makes sure that there are no $k$ such that $i \leq k \leq c_{1}$ and $j \leq w(k) \leq w\left(c_{1}\right)$ except $k=c_{1}$ or in other words, there are no dots (permutation entries) strictly inside the rectangle from $(i, j)$ to $\left(c_{1}, w\left(c_{1}\right)\right)$ in the Rothe diagram $R D(w)$.

We will now construct a special RC-graph $D_{(i, j)} \in \mathcal{R C}(w)$ via simple ladder moves from $B_{w}$ such that non-simple ladder moves are ready to be applied to $D_{(i, j)}$. Let $I=\left\{i^{\prime} \mid i \leq i^{\prime} \leq c_{1},\left(i^{\prime}, j\right) \in R D(w)\right\}$ and $J=\left\{j^{\prime} \mid j \leq j^{\prime} \leq w\left(c_{1}\right),\left(i, j^{\prime}\right) \in\right.$ $R D(w)\}$. Since there are no permutation entries in the rectangle in $R D(w)$ from $(i, j)$ to $\left(c_{1}, w\left(c_{1}\right)\right)$, all squares inside this region are exactly $I \times J \backslash\left\{\left(c_{1}, w\left(c_{1}\right)\right)\right\}$. See Figure [6 for an example of these squares. Let $I=\left\{i=i_{0}<i_{1}<\cdots<i_{r}=c_{1}\right\}$, $r \geq 1$ and $J=\left\{j=j_{0}<j_{1}<\cdots<j_{m}=w\left(c_{1}\right)\right\}, m \geq 1$. For each $i_{k} \in I$, in increasing order of $k$ from 0 to $r$, and for each $j^{\prime} \geq j$ such that $\left(i_{k}, j^{\prime}\right) \in R D(w)$ (not just $j^{\prime} \in J$ ) in decreasing order of $j^{\prime}$, apply a simple ladder move $i_{k}-i-k$ times in $B_{w}$ to $\beta\left(i_{k}, j^{\prime}\right)$. Let this RC-graph be $D_{(i, j)}^{(0)}$. See Figure 6 for an example. Intuitively, from $B_{w}$, we apply simple ladder moves to row $i_{k}$ starting from the crossing $\beta\left(i_{k}, j\right)$ enough times such that the squares $I \times J \backslash\left\{\left(c_{1}, w\left(c_{1}\right)\right)\right\} \subset R D(w)$ form a consecutive rectangle without a corner in $D_{(i, j)}^{(0)}$. To see that such process is possible, it suffices to show that $(s, t) \in R D(w)$ won't interfere these simple 
moves, for some $i \leq s \leq c_{1}$. If $s \notin I$, by definition of $I, w(s)<j$ so $t<j$. Then for $\left(i^{\prime}, j^{\prime}\right) \in I \times J \backslash\left\{c_{1}, w\left(c_{1}\right)\right\}$, if $i^{\prime}<s$, clearly $(s, t)$ won't be in the way since $\beta\left(i^{\prime}, j^{\prime}\right)$ are moving up by simple ladder moves but $(s, t)$ is lower (at a larger row number) to start with, and if $i^{\prime}>s$, with $j^{\prime} \geq j>t$, by Lemma 2.5. we are done as well. The case $s \in I$ is argued in the exact same way. Recall that if $\left(i^{\prime}, j^{\prime}\right) \in R D(w)$ and $\left(i^{\prime \prime}, j^{\prime \prime}\right) \in R D(w)$ are in the same row (or column) and there are no other boxes in between, then in $B_{w}$, the diagonals where they are on differ by exactly 1 . As a result, from $B_{w}$ to $D_{(i, j)}^{(0)}$, we have moved $\beta\left(I \times J \backslash\left\{\left(c_{1}, w\left(c_{1}\right)\right)\right\}\right)$ to $\{i, i+1, \ldots, i+r\} \times\{l, l+1 \ldots, l+m\} \backslash\{(i+r, l+m)\}$ where $\beta(i, j)=(i, l)$.
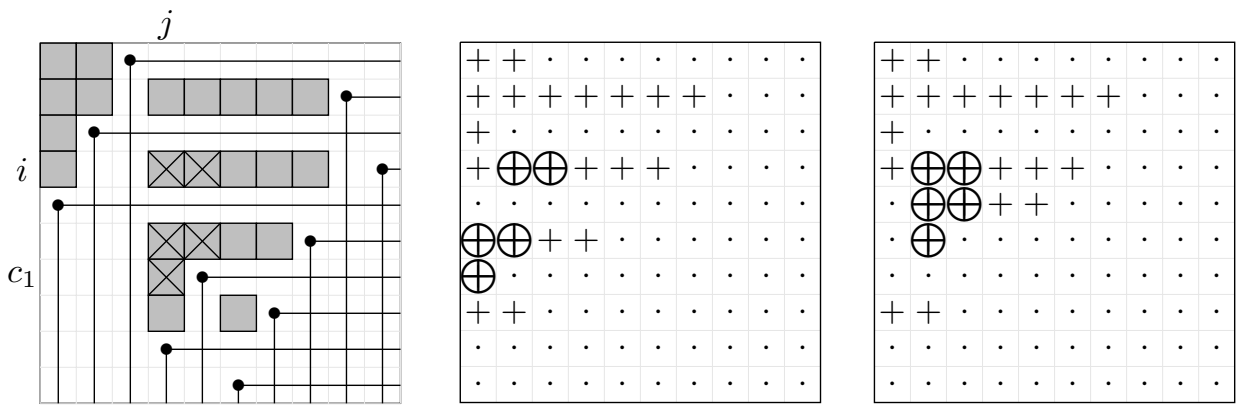

Figure 6 . The Rothe diagram $R D(w)$, the bottom RC-graph $B_{w}$ and $D_{(i, j)}^{(0)}$ for $w=3,9,2,10,1,8,5,7,4,6$, where $(i, j)=(4,4)$, $\# A_{(i, j)}=2, \# C_{(i, j)}=3, c_{1}=7, I=\{4,6,7\}, J=\{4,5\}$ and squares $I \times J \backslash\left\{\left(c_{1}, w\left(c_{1}\right)\right)\right\}$ are marked.

From $D_{(i, j)}^{0}$, for each $i^{\prime}<i$ in increasing order and for each $j^{\prime} \geq j$ in decreasing order, we apply simple ladder moves $\#\left\{a<i^{\prime} \mid w(a)<j\right\}$ times to $\beta\left(i^{\prime}, j^{\prime}\right)$ if $\left(i^{\prime}, j^{\prime}\right) \in R D(w)$. Here we abuse the notation of $\beta(-,-)$ to also mean squares in $D_{(i, j)}^{(0)}$ since previously we moved squares after row $i$ and now we move squares before row $i$. Call this new RC-graph $D_{(i, j)}$. The purpose of this step is to make sure that the key squares $\{i, \ldots, i+r\} \times\{l, \ldots, l+m\} \backslash\{(i+r, l+m)\}$ in $D_{(i, j)}$ have enough room above row $i$ to move around. See Figure 7 as a continuing example from Figure 6]. More formally, for $\left(i^{\prime}, j^{\prime}\right) \in R D(w)$ with $i^{\prime}<i$, if $j^{\prime}<j$, then this square has never moved from $B_{w}$ to $D_{(i, j)}$ and by Lemma 2.5, $\operatorname{diag}\left(\beta\left(i^{\prime}, j^{\prime}\right)\right)-$ $\operatorname{diag}(\beta(i, j)) \leq-2$ and if $j^{\prime} \geq j$, then its corresponding square in $D_{(i, j)}$ has a row number at most $i^{\prime}-\#\left\{a<i^{\prime} \mid w(a)<j\right\} \leq i-1-\# A_{(i, j)}$. As a result, $\left(i^{\prime}, j^{\prime}\right) \notin D_{(i, j)}$ for all $i-\# A_{(i, j)} \leq i^{\prime} \leq i-1$ and $j^{\prime} \geq(i+j-1)-i^{\prime}$. These important empty squares are also marked in the running example in Figure 7

As a summary, we have $\{i, \ldots, i+r\} \times\{l, \ldots, l+m\} \backslash\{(i+r, l+m)\} \subset D_{(i, j)}$, $(i+r, l+m) \notin D_{(i, j)}$ and $\left(i^{\prime}, j^{\prime}\right) \notin D_{(i, j)}$ for all $i-\# A_{(i, j)} \leq i^{\prime} \leq i-1$ and $j^{\prime} \geq$ $(i+j-1)-i^{\prime}$. Recall $C_{(i, j)}=\left\{c_{1}, \ldots, c_{q}\right\}$ and each permutation entry $c_{2}, \ldots, c_{q}$ will create a box in row $i$ so we also have $(i, l+m+1), \ldots,\left(i, l+m+q_{0}\right) \in D_{(i, j)}$ for some $q_{0} \geq q-1$ with $\left(i, l+m+q_{0}+1\right) \notin D_{(i, j)}$. We can now construct $\mathcal{P}_{(i, j)} \subset \mathcal{R C}(w)$ of cardinality $\# A_{(i, j)} \cdot \# C_{(i, j)}$. Choose any $0 \leq a^{\prime}<\# A_{(i, j)}$ and $0 \leq c^{\prime}<\# C_{(i, j)}=q$. From $D_{(i, j)}$, applying ladder moves of order $r$ to crossings $(i+r, l+m-1), \ldots,(i+r, l)$ in such order to obtain crossings $(i-1, l+m), \ldots,(i-1, l+1)$, applying simple ladder moves to crossings $\left(i, l+m+q_{0}\right), \ldots,\left(i, l+m+q_{0}-c^{\prime}+1\right)$ to obtain crossings 

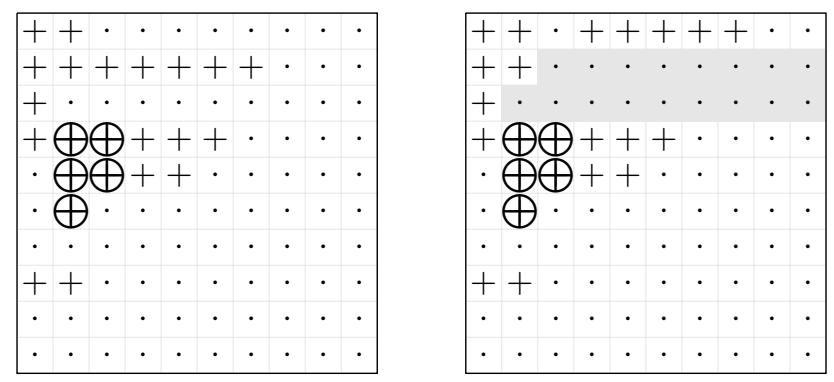

Figure 7. The RC-graph $D_{(i, j)}^{(0)}$ and $D_{(i, j)}$ for $w=$ $3,9,2,10,1,8,5,7,4,6$ and $(i, j)=(4,4)$ where important empty space is shaded.

$\left(i-1, l+m+q_{0}+1\right), \ldots,\left(i-1, l+m+q_{0}-c^{\prime}+2\right)$ and applying simple ladder moves $a^{\prime}$ times to these newly obtained crossings together (from right to left) result in $D_{(i, j)}^{\left(a^{\prime}, c^{\prime}\right)}$. Notice that $l+m+q_{0}-c^{\prime}+2$ is at least 2 greater than $l+m$ and that there are at least $\# A_{(i, j)}$ empty rows above them at diagonal number $\geq i+l-1$ so these moves are possible. See Figure 8 for an example.
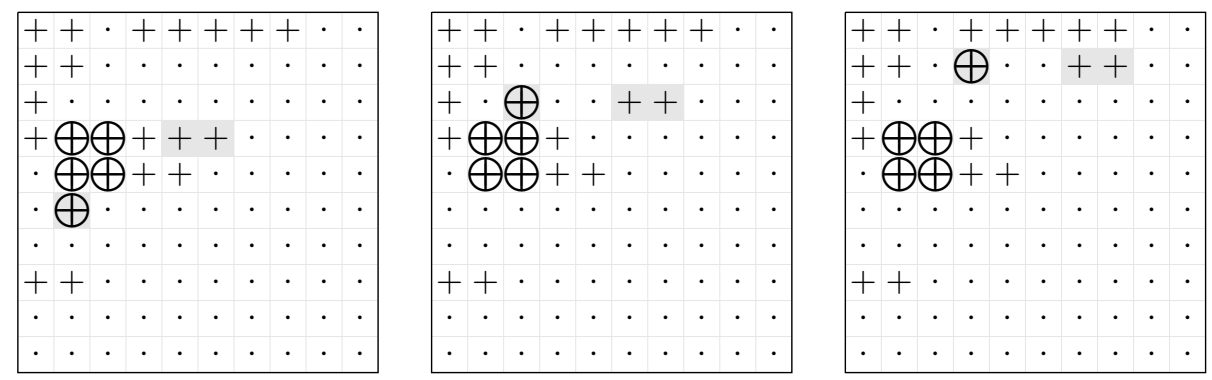

Figure 8. The RC-graph $D_{(i, j)}, D_{(i, j)}^{(0,2)}$ and $D_{(i, j)}^{(1,2)}$ for $w=$ $3,9,2,10,1,8,5,7,4,6,(i, j)=(4,4)$ where the crossings moved are shaded.

We see that $\# \mathcal{P}_{(i, j)}=\# A_{(i, j)} \cdot \# C_{(i, j)}$ so it remains to show that these RC-graphs are distinct for different $(i, j)$ 's. Let $D$ be an RC-graph that is in some $\mathcal{P}_{(i, j)}$ and we will show that we can recover $(i, j)$ from $D$. Compare $D$ with $B_{w}$. Recall that a crossing has type $(a, b)$ if strand $a$ and strand $b$ intersects at this crossing. Let $X$ be the set of inversions of $w$ such that the crossing in $D$ with type $(a, b)$ has different diagonal number than the crossing in $B_{w}$ with type $(a, b)$. Notice that simple ladder moves do not change the diagonal number of a crossing of a fixed type. By the construction illustrated above, the crossings in $D$ with types in $X$ must all be in the same row and are consecutive. Let them be $\left(i^{\prime}, j^{\prime}\right), \ldots,\left(i^{\prime}, j^{\prime \prime}\right)$. Let $i$ be the smallest integer such that $i>i^{\prime}$ and $\left(i, i^{\prime}+j^{\prime}-i\right) \in D$. Then $(i, j) \in R D(w)$ is the box that corresponds to $\beta(i, j)=\left(i, i^{\prime}+j^{\prime}-i\right) \in B_{w}$. 


\section{The MAin CONJECTURE}

As Theorem 2.1 strengthens Theorem 2.2, it is natural to ask how far we can push the results of this form. In this section, we provide our main conjecture which suggests that there is strong relation between $\mathfrak{S}_{w}(1)$ and patterns contained in $w$.

We define a sequence of integers $\left\{c_{u}\right\}_{m \geq 1, u \in S_{m}}$ recursively:

$$
c_{w}:=\mathfrak{S}_{w}(1)-1-\sum_{|u|<|w|} c_{u} p_{u}(w)
$$

where $|u|=m$ if $u \in S_{m}$.

In other words, we have $\mathfrak{S}_{w}(1)=1+\sum_{|u| \leq|w|} c_{u} p_{u}(w)$.

Lemma 3.1. For $w \in S_{n}$ with $w(n)=n, c_{w}=0$.

Proof. We prove the claim by induction. When $w=$ id is the identity permutation, $\mathfrak{S}_{\text {id }}(1)=1$ so $c_{\text {id }}=0$. Now assume that $w(n)=n$ with $w \in S_{n}, n \geq 2$ and let $w^{\prime} \in S_{n-1}$ be the permutation such that $w^{\prime}(i)=w(i)$ for all $i=1, \ldots, n-1$. The stability property of Schubert polynomials implies that $\mathfrak{S}_{w^{\prime}}(1)=\mathfrak{S}_{w}(1)$. We also observe that for $u \in S_{k}$ with $k \leq n-1, p_{u}\left(w^{\prime}\right)=p_{u}(w)$ except $u(k)=k$, in which case $c_{u}=0$ by induction hypothesis. Thus,

$$
\begin{aligned}
c_{w} & =\mathfrak{S}_{w}(1)-1-\sum_{|u| \leq n-1} c_{u} p_{u}(w) \\
& =\mathfrak{S}_{w^{\prime}}(1)-1-\sum_{|u| \leq n-1} c_{u} p_{u}\left(w^{\prime}\right) \\
& =\mathfrak{S}_{w^{\prime}}(1)-1-\sum_{|u| \leq n-2} c_{u} p_{u}\left(w^{\prime}\right)-\sum_{|u|=n-1} c_{u} p_{u}\left(w^{\prime}\right) \\
& =c_{w^{\prime}}-c_{w^{\prime}} p_{w^{\prime}}\left(w^{\prime}\right)=0 .
\end{aligned}
$$

Lemma 3.1 allows us to define $c_{w}$ for $w \in S_{\infty}$. The first few $w \in S_{\infty}$ with nonzero values $c_{w}$ are $c_{132}=1, c_{1432}=1$ followed by 23 permutation patterns in $S_{5}$.

Conjecture 3.2. We have $c_{w} \geq 0$ for all $w \in S_{\infty}$.

Conjecture 3.2 has been verified by computer for all $w \in S_{n}, n \leq 8$. Notice that Conjecture 3.2 immediately implies Theorem 2.1 but only checking $c_{1432}=1$ would not be enough to conclude Theorem 2.1. Computational evidence also seems to suggest that for a fixed $n$, the permutations $w \in S_{n}$ that achieve maximum are layered. They are listed in Table 1. This list of permutations is almost identical (except $n=5$ ) to that of the permutations achieving maximum values at $\mathfrak{S}_{w}(1)$ ([5], [6]). It is natural to conjecture that these two lists are the same when $n \geq 6$.

Certain values of $c_{w}$ 's can be easily computed. First, if $w$ avoids 132 then all of its patterns avoid 132 and it is well-known that $\mathfrak{S}_{w}(1)=1$. So we know $c_{w}=0$ by induction. Let $w^{(n)} \in S_{n}$ denote the permutation $1, n, n-1, \ldots, 2$. We know that $\mathfrak{S}_{w^{(n)}}=C_{n-1}$, the $(n-1)^{t h}$ Catalan number [8]. Such $w^{(n)}$ can only contain a permutation $w^{(m)}$, for some $m \leq n$ or $u=m, m-1, \ldots, 1$ for which $c_{u}=0$. A straightforward calculation using induction produces the constants $c_{w^{(n)}}$ for $n \geq 3$ and this sequence is recorded as A005043 in OEIS. 


\begin{tabular}{c|c|c}
$n$ & $\max c_{w}$ & $w$ \\
\hline 3 & 1 & 132 \\
4 & 1 & 1432 \\
5 & 5 & 12543,21543 \\
6 & 37 & 126543,216543 \\
7 & 342 & 1327654 \\
8 & 5820 & 13287654
\end{tabular}

TABLE 1. The maximum value of $c_{w}$ and those permutations $w \in$ $S_{n}$ that achieve this value for $n \leq 8$.

Remark 3.3. Conjecture 3.2 was observed independently by Christian Gaetz while this paper is in the writing process.

\section{Simple ladder moves and 1432 aVoiding Permutations}

In this section, we explore more regarding simple ladder moves on RC-graphs. The main theorem of this section is the following.

Theorem 4.1. The following two conditions for $w \in S_{\infty}$ are equivalent:

(1) any two $R C$-graphs of $w$ are connected by simple ladder moves;

(2) $w$ avoids 1432.

Remark 4.2. If $w$ avoids 321, which is a stronger condition than $w$ avoiding 1432, then $w$ is fully commutative: any two reduced expressions for $w$ are connected by commutator moves $s_{i} s_{j}=s_{j} s_{i}$ for $|i-j| \geq 2$, meaning that there are no Coxeter moves $s_{i} s_{i+1} s_{i}=s_{i+1} s_{i} s_{i+1}$ available for any reduced expression of $w$. This stronger condition immediately implies (1) of Theorem 4.1 since a non-simple ladder move changes how many simple transpositions $s_{i}$ 's are used (or equivalently, the label of $D$ ) in the reduced expression $\prod_{i=1}^{\infty} \prod_{j=\infty,(i, j) \in D}^{1} s_{i+j-1}$ of $w$ corresponding to $D \in \mathcal{R C}(w)$.

We start with some observations on simple ladder moves on RC-graphs. Let $D \in \mathcal{R C}(w)$. Consider all positions on diagonal $k$ and $k+1$ in the following order from lower left to upper right: $(k+1,1),(k, 1),(k, 2),(k-1,2), \ldots,(1, k),(1, k+$ $1)$. Recall that a simple ladder move at coordinate $(a, b)$ require that $(a, b) \in D$, $(a-1, b),(a, b+1),(a-1, b+1) \notin D$ and it changes $(a, b)$ to $(a-1, b+1)$, staying on the same diagonal. Therefore, for all crossings in $D$ on diagonal $k$ and $k+1$, their relative positions in this ordering will never change after simple ladder moves. Notice that each crossing is labeled uniquely so we can identify the same crossing in different RC-graphs.

Lemma 4.3. Let $D \in \mathcal{R C}(w)$ be obtained from $B_{w}$ after a sequence of ladder moves. If $(i, j),(i, j+1) \in D$, then their corresponding crossings (crossings with the same label) in $B_{w}$ are adjacent in the same row as well.

Proof. Recall that crossings of $B_{w}$ are left-justified: on row $i$, the crossings in $B_{w}$ are exactly $(i, 1), \ldots,\left(i, \operatorname{code}(w)_{i}\right)$. Let $k=i+j-1$ so that $\operatorname{diag}(i, j)=k$ and $\operatorname{diag}(i, j+1)=k+1$. Consider the chain of all positions on diagonal $k$ and $k+1$ : $(k+1,1),(k, 1), \ldots,(1, k),(1, k+1)$. We see that in $D,(i, j)$ appears before $(i, j+1)$ and that there are no other crossings in between. Let $(a, b),\left(a^{\prime}, b^{\prime}\right) \in B_{w}$ be the corresponding crossings to $(i, j),(i, j+1) \in D$ respectively. With the observation 
above, in the chain of all positions on diagonal $k$ and $k+1,(a, b)$ must appear before $\left(a^{\prime}, b^{\prime}\right)$ and that there are no crossings in between. If $b^{\prime}=1$, then $a^{\prime}=k+1$ so $\left(a^{\prime}, b^{\prime}\right)$ is the start of this chain and there are no positions available for $(a, b)$. Thus $b^{\prime} \neq 1$. Then $\left(a^{\prime}, b^{\prime}-1\right) \in B_{w}$ which implies $\left(a^{\prime}, b^{\prime}-1\right)=(a, b)$ so we are done.

We point out that simple ladder moves satisfy the "diamond condition" and thus have "local confluence". Specifically, for $D \in \mathcal{R C}(w)$, if we can apply simple ladder moves at two different crossings $\left(i_{1}, j_{1}\right) \in D$ and $\left(i_{2}, j_{2}\right) \in D$, resulting in two RCgraphs $D_{1}$ and $D_{2}$ respectively. Then we can apply a simple ladder move at $\left(i_{2}, j_{2}\right) \in$ $D_{1}$ and $\left(i_{1}, j_{1}\right) \in D_{2}$, resulting in the same RC-graph. This "diamond property" is easy to verify. The following diamond lemma is very commonly seen in the theory of chip-firing, which is used to show that the resulting stable configuration does not depend on the firing sequence.

Lemma 4.4 (Diamond lemma). Let $G$ be a directed graph with a unique source $s$ such that for any vertex $v$ with at least two outgoing edges $v \rightarrow v_{1}, v \rightarrow v_{2}$, there exists some other vertex $v^{\prime}$ such that $v_{1} \rightarrow v^{\prime}$ and $v_{2} \rightarrow v^{\prime \prime}$. Then for any vertex $v$, all paths from $s$ to $v$ have the same length. Moreover, either $G$ is infinite and does not have a sink or $G$ has a unique sink.

The proof of Lemma 4.4 is left as an exercise.

In our scenario, $B_{w}$ is the source that generates a directed graph via simple ladder moves. The unique sink is $T_{w}$ since it is shown that there exists some sequence of simple ladder moves taking $B_{w}$ to $T_{w}$ [7, and that ladder move is applicable to $T_{w}$ because $T_{w}$ is top-justified. With the help of the diamond lemma, we immediately know that starting from $B_{w}$, if we apply any sequence of simple ladder moves until no simple ladder moves are available, then we arrive at $T_{w}$.

We can rephrase Lemma 4.3 as follows. Let $D \in \mathcal{R C}(w)$ be obtained from $B_{w}$ via simple ladder moves, or equivalently, obtained from $T_{w}$ via inverse simple ladder moves. If $(i, j),(i, j+1) \in D$, then their corresponding boxes in $R D(w)$ lie in the same row and that there are no other boxes in between. Dually, if $(i, j),(i+1, j) \in D$, then their corresponding boxes in $R D(w)$ lie in the same column and that there are no other boxes in between.

Proof of Theorem 4.1. Recall that simple ladder moves don't change the label of an RC-graph while non-simple ladder moves increase the label lexicographically. We also know that all RC-graphs of $w$ can be obtained from $B_{w}$ by ladder moves. Therefore, it suffices to show that $w$ contains 1432 if and only if we can apply a non-simple ladder move to some RC-graph of $w$. Therefore, if $w$ contains 1432, the proof of Theorem 2.1 produces an RC-graph obtained from $B_{w}$ via simple ladder moves and at least one non-simple ladder move. This shows $(1) \Rightarrow(2)$.

The main case of this theorem is $(2) \Rightarrow(1)$. Assume the opposite that $w$ avoids 1432 and that we can apply a non-simple ladder move to an RC-graph $D \in \mathcal{R C}(w)$. It suffices to consider the case that $D$ is obtained from $B_{w}$ via a sequence of simple ladder moves. Assume that $\left(i^{\prime}, j^{\prime}\right),\left(i^{\prime}+1, j^{\prime}\right), \ldots,\left(i^{\prime}+k, j^{\prime}\right) \in D,\left(i^{\prime}, j^{\prime}+1\right), \ldots,\left(i^{\prime}+\right.$ $\left.k-1, j^{\prime}+1\right) \in D$ and $\left(i^{\prime}+k, j^{\prime}+1\right) \notin D$ so that a ladder move of order $k$ can be applied to $\left(i^{\prime}+k-1, j^{\prime}+1\right)$ in $D$. Here $k \geq 1$. For these $2 k+1$ crossings in $D$, consider their corresponding boxes in $R D(w)$. By Lemma 4.3, there exists $I=\left\{i_{0}<\cdots<i_{k}\right\}$ and $J=\left\{j_{1}<j_{2}\right\}$ such that these corresponding boxes are $I \times J \backslash\left\{\left(i_{k}, j_{2}\right)\right\}$. As a basic property of Rothe diagrams, if $(a, b) \in R D(w)$ and 
$\left(a^{\prime}, b^{\prime}\right) \in R D(w)$ with $a<a^{\prime}$ and $b>b^{\prime}$, then $\left(a, b^{\prime}\right) \in R D(w)$. Together with the fact from Lemma 4.3 that there are no other boxes in between $\left(i_{a}, j_{1}\right)$ and $\left(i_{a}, j_{2}\right)$ for $a=0, \ldots, k$ or $\left(i_{a}, j_{b}\right)$ and $\left(i_{a+1}, j_{b}\right)$ for $b=1,2$ and $a=0, \ldots, k-1$, we conclude that $I \times J \backslash\left\{\left(i_{k}, j_{2}\right)\right\}$ are the only squares with coordinates in $\left\{i_{0}, \ldots, i_{k}\right\} \times\left\{j_{1}, j_{2}\right\} \backslash$ $\left\{\left(i_{k}, j_{2}\right)\right\}$. See Figure 9 for an example of these boxes.

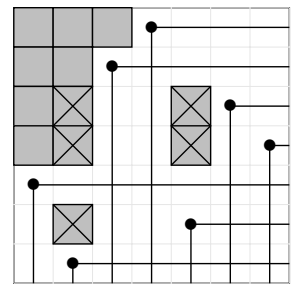

FiguRE 9. Rothe diagram of $w=4367152$ which avoids 1432, with $I \times J \backslash\left\{i_{k}, j_{2}\right\}$ marked.

We then claim that $\left(i_{k}, j_{2}\right) \notin R D(w)$ and in fact $w\left(i_{k}\right)=j_{2}$. Recall that $\left(i_{k-1}, j_{2}\right) \in R D(w)$ corresponds to $\left(i^{\prime}+k-1, j^{\prime}+1\right) \in D$ and $\left(i_{k}, j_{1}\right) \in R D(w)$ corresponds to $\left(i^{\prime}+k, j^{\prime}\right) \in D$, which lie on diagonal $i^{\prime}+j^{\prime}+k-1$. Consider the chain of positions on diagonals $i^{\prime}+j+k-1$ and $i^{\prime}+j+k$. Since $\left(i^{\prime}+k, j^{\prime}+1\right) \notin D$, $\left(i^{\prime}+k, j^{\prime}\right)$ and $\left(i^{\prime}+k-1, j^{\prime}+1\right)$ are adjacent in this chain. If $\left(i_{k}, j_{2}\right) \in R D(w)$, then its corresponding crossing in $D$ sits in between, which is a contradiction. As a result $\left(i_{k}, j_{2}\right) \notin R D(w)$. This means either $j_{1}<w\left(i_{k}\right)<j_{2}$, which is impossible since it would imply $\left(i_{0}, w\left(i_{k}\right)\right) \in R D(w)$ lying between $\left(i_{0}, j_{1}\right)$ and $\left(i_{0}, j_{2}\right)$, or $i_{k-1}<w^{-1}\left(j_{2}\right)<i_{k}$, which is impossble for the same reason, or $w\left(i_{k}\right)=j_{2}$, which is only possibility.

As a summary, we see that $\left(i_{0}, j_{1}\right) \in R D(w)$ and $w\left(i_{k}\right)=j_{2}$. So $i_{0}<i_{k}<$ $w^{-1}\left(j_{1}\right)$ and $w\left(i_{0}\right)>w\left(i_{k}\right)>j_{1}$. As $w$ avoids 1432 , there does not exist $a<i_{0}$ such that $w(a)<j_{1}$. As a result, $(a, b) \in R D(w)$ for all $a \leq i_{0}$ and $b \leq j_{1}$. After left-justification to $B_{w}$, we have $(a, b) \in B_{w}$ for all $a \leq i_{0}$ and $b \leq j_{1}$ as well. These crossings will always be there after any sequence of simple ladder moves. As a result, $\left(i_{0}, j_{1}\right) \in R D(w)$ corresponds to $\left(i_{0}, j_{1}\right) \in B_{w}$ and the same box in $\left(i^{\prime}, j^{\prime}\right) \in D$. However, $\left(i^{\prime}-1, j^{\prime}\right) \in D$ as well, contradicting the condition for a ladder move.

\section{ACKNOWLEDGEMENTS}

The author thanks Christian Gaetz, Alex Postnikov and Richard Stanley for helpful conversations.

\section{REFERENCES}

[1] Nantel Bergeron and Sara Billey. RC-graphs and Schubert polynomials. Experiment. Math., 2(4):257-269, 1993.

[2] Allen Knutson and Ezra Miller. Gröbner geometry of Schubert polynomials. Ann. of Math. (2), 161(3):1245-1318, 2005.

[3] Laurent Manivel. Symmetric functions, Schubert polynomials and degeneracy loci, volume 6 of $S M F / A M S$ Texts and Monographs. American Mathematical Society, Providence, RI; Société Mathématique de France, Paris, 2001. Translated from the 1998 French original by John R. Swallow, Cours Spécialisés [Specialized Courses], 3. 
[4] Grigory Merzon and Evgeny Smirnov. Determinantal identities for flagged Schur and Schubert polynomials. Eur. J. Math., 2(1):227-245, 2016.

[5] Alejandro H. Morales, Igor Pak, and Greta Panova. Asymptotics of principal evaluations of Schubert polynomials for layered permutations. Proc. Amer. Math. Soc., 147(4):1377-1389, 2019.

[6] Richard P Stanley. Some schubert shenanigans. arXiv preprint arXiv:1704.00851, 2017.

[7] Anna E. Weigandt. Schubert polynomials, 132-patterns, and Stanley's conjecture. Algebr. Comb., 1(4):415-423, 2018.

[8] Alexander Woo. Catalan numbers and schubert polynomials for $w=1(n+1) \ldots 2$. arXiv preprint math/0407160, 2004.

Department of Mathematics, Massachusetts Institute of Technology, Cambridge, Ma 02139 E-mail address: gaoyibo@mit.edu 